\title{
Differential gene expression in roots of yellow lupin sprouts under Fusarium treatment
}

\author{
Sysoliatin E.N. ${ }^{*}$, Anisimova N.A. ${ }^{1}$, Anokhina V.S. ${ }^{2}$, Kilchevsky A.V. ${ }^{1}$ \\ ${ }^{1}$ Institute of Genetics and Cytology, NASB, Minsk, Belarus \\ ${ }^{2}$ Belarusian State University, Minsk, Belarus \\ *e-mail:Meeugeny@yandex.ru
}

Motivation and Aim: Yellow lupin (Lupinus luteus L.) is a valuable high-protein legume plant. Nevertheless, its implementation as field crop is restricted by heavy damage caused by fungal pathogens. One of the most devastating disease for yellow lupin is fusariosis. The aim of this work was to assess the feasibility of using SRAP (sequencerelated amplified polymorphism) for transcriptome profiling analysis of yellow lupine samples under Fusarium treatment.

Methods and Algorithms: RNA was isolated from the roots of ten-day seedlings of yellow lupine varieties Tremosilla, BSHA 13, BSHA 19, Nadezhny. There were four groups of seedlings: intact and exposed to three Fusarium isolates. cDNA was synthesized on the RNA matrix and was used in a series of PCR reactions with 12 combinations of 3 forward (Me8, f12, f16) and 4 reverse (Em5, Em12, r14, r9) SRAP primers. SRAP fragments associated with resistant seedlings were isolated and sequenced. Their putative function was assigned based on alignment to known sequences.

Results: The results showed that 12 primer combinations produced a total of 107 clear bands. The proportion of polymorphic bands varied in the range from $81.3 \%$ to $100 \%$. Three SRAP fragments were associated with longer roots (f12-Em5-120, f16-r9-500) and hypocotyl (f12-Em5-200) in seedlings treated with Fusarium isolates. Sequencing and subsequent analysis of these fragments allowed to associate fragment f12-Em5-120 with fumarylacetoacetase mRNA, f16-r9-500 with BURP domain protein USPL1-like mRNA, f12-Em5-200 with hydroxyphenylpyruvate dioxygenase mRNA.

Conclusion: The results obtained indicate that the SRAP method is suitable for the analysis of yellow lupine cDNA and can be used to study the differential gene expression of this culture. Three SRAP fragmets associated with mRNA expressed in Fusarium treated seedlings were found. 